\title{
PAX8 is a potential marker for the diagnosis of primary epithelial ovarian cancer
}

\author{
HONG-JUAN CHAI ${ }^{1 *}$, QING REN ${ }^{1 *}$, QIN FAN ${ }^{1}$, LIU YE ${ }^{1}$, GUANG-YE DU ${ }^{2}$, \\ HUA-WEN DU ${ }^{1}$, WEI XU ${ }^{1}$, YUE LI ${ }^{1}$, LAN ZHANG ${ }^{1}$ and ZHONG-PING CHENG ${ }^{1,2}$ \\ ${ }^{1}$ Department of Gynecology and Obstetrics, Shanghai Ninth People's Hospital, Shanghai Jiaotong \\ University School of Medicine, Shanghai 201999; ${ }^{2}$ Department of Gynecology and Obstetrics, \\ Yangpu Hospital, School of Medicine, Tongji University, Shanghai 200090, P.R. China
}

Received August 9, 2015; Accepted December 9, 2016

DOI: $10.3892 / 01.2017 .6949$

\begin{abstract}
The present study aimed to investigate the clinical significance of paired-box 8 (PAX8) in primary epithelial ovarian cancer (PEOC). Using immunohistochemical (IHC) staining, the expression of PAX8 in 60 patients with PEOC, 20 patients with ovarian benign lesions and 10 patients with metastatic ovarian cancer (MOC), was examined based on the clinicopathological profiles of the patients. The correlation between PAX8 expression and the clinicopathological parameters or prognosis of patients was statistically analyzed. PAX8 was revealed to be highly expressed in PEOC, but not in MOC, as indicated by IHC staining. The rate of positivity of PAX8 in PEOC was 92\% (57/60) with no significant difference of PAX8 expression found between the various pathological types of PEOC $(\mathrm{P}=0.871)$. The rate of positivity of PAX8 in ovarian benign tumors was $85 \%$, demonstrating no significant difference in comparison with that of PEOC $(\mathrm{P}=0.761)$. PAX8 staining and statistical analysis revealed that the higher the grade of PEOC, the less the cancer cell had differentiated $(\mathrm{P}=0.033)$ and the more the cancer had advanced according to International Federation of Gynaecological Oncologists (FIGO) staging $(\mathrm{P}=0.003)$. Survival rate statistics showed that PEOC patients with higher PAX8 expression exhibited a shorter postoperative survival rate $(\mathrm{P}=0.009)$. $\mathrm{PAX} 8$ was specifically expressed in PEOC, and its expression level was associated with the degree of cancer cell differentiation, FIGO stage, and survival rate, indicating that PAX8 is a potential marker for the diagnosis of PEOC.
\end{abstract}

Correspondence to: Professor Zhong-Ping Cheng, Department of Gynecology and Obstetrics, Shanghai Ninth People's Hospital, Shanghai Jiaotong University School of Medicine, 280 Mohe Road, Shanghai 201999, P.R. China

E-mail: mdcheng18@263.net

*Contributed equally

Key words: paired-box 8, primary epithelial ovarian cancer, metastatic ovarian cancer, prognosis

\section{Introduction}

Ovarian cancer (OC) is the most lethal of all types of gynecological malignancy and the third leading cause of cancer mortality among females worldwide (1). Due to the lack of specific biomarkers for the diagnosis, more than half of patients are found to be in the advanced stage of OC at the time of initial diagnosis $(2,3)$. Among the multiple types of ovarian cancer that exist, primary epithelial ovarian cancer (PEOC) accounts for $85-90 \%$ in total (4). Another type, metastatic ovarian carcinoma (MOC), can form from gastrointestinal tumors and breast cancers with peritoneal dissemination and/or lymphatic metastasis (5-7).

The clinical use of cancer antigen (CA)-125, estrogen receptor (ER) and progesterone receptor (PR) for the diagnosis of OC is currently not effective due to low sensitivity and specificity $(8,9)$. Therefore, the screening and identification of a specific biomarker for OC diagnosis is important. In a previous study, paired-box 8 (PAX8) has been suggested to be a sensitive marker for tumors of the thyroid, kidney and thymus, and tumors derived from the Müllerian pipe organ (10-12). It was also reported that PAX8 is widely expressed in various types of ovarian tumors, particularly in serous tumors such as ovarian endometrioid carcinoma and clear cell carcinoma (11). Through the collating, summarizing and long-term follow-up inspection of preliminary clinical data on ovarian cancer, the present study mainly discusses the value of PAX8 in the differential diagnosis of primary and metastatic ovarian carcinoma, and analyzes the association between the expression of PAX8, clinicopathological features of primary ovarian carcinoma and prognosis for patients with ovarian cancer.

\section{Materials and methods}

Research subjects. The present study was approved by the hospital Review Board and the Ethics Committee of the Medical Faculty at the Shanghai Jiao Tong University (Shanghai, China). All patients provided written informed consent of using their tumor tissues prior to the beginning of the study. In total, 60 PEOC patients enrolled in the Gynecology and Obstetrics Department of the Shanghai Ninth People's Hospital (Shanghai, China), whom underwent 
radical resection between January 2008 and December 2012, were included in the present study. The age of patients ranged between 39 and 68 years, with a median age of 52 . The group included 44 patients with ovarian serous carcinoma, 9 patients with mucinous carcinoma, 2 patients with endometrial carcinoma and 5 patients with clear cell carcinoma. The control group included 20 patients with benign ovary tumors and 10 patients with MOC. In the MOC subgroup, the MOC had metastasized in 5 patients from gastric cancer, from colon cancer in 3 patients and from breast cancer in 2 patients. All clinicopathological profiles were evaluated in accordance with the criteria of the National Comprehensive Cancer Network Clinical Practice Guidelines in Oncology (Fort Washington, PA, USA) (3). None of the patients received preoperative radiotherapy or chemotherapy prior to admission to hospital, confirmed by pathology following the surgery, and all were treated with platinum-based chemotherapy in combination with taxol subsequent to the surgery.

The last follow-up was on December 31, 2014, with an average follow-up time of $20.58 \pm 5.71$ months. During the follow-up period, 45 patients succumbed to recurrence or metastasis. The remaining 15 patients had censored data (among which 3 succumbed to other diseases, 2 were lost to follow-up and 10 are still alive). Surviving patients had a minimum follow-up period of 16 months, with the longest being 36 months.

Immunohistochemical (IHC) staining. Surgically-obtained tumor tissue specimens were prepared in the form of $4 \mu \mathrm{m}$ thick sections, prepared with hematoxylin and eosin staining and IHC staining, respectively. The avidin-biotin complex (ABC) method of IHC staining was adopted. The sections were treated by normal dewaxing, graded ethanol hydration, $3 \% \mathrm{H}_{2} \mathrm{O}_{2}$ closed endogenous peroxidase, microwave heating antigen retrieval and PBS rinsing. The primary antibody used was an anti-PAX8 rabbit anti-human monoclonal antibody (dilution 1:200; \#ab189249; Abcam, Cambridge, UK) and the biotinylated secondary antibody was a goat anti-rabbit antibody (dilution, 1:200; \#ab7010; Abcam). ABC reagent was used to visualize the staining (ABC kit; Santa Cruz Biotechnology, Inc., Dallas, TX, USA) and samples were subsequently stained with 3,3'-diaminobenzidine. Next, the samples were re-stained with hematoxylin and were resin-sealed. A PAX8 positive result was taken to be the nucleus appearing yellowish-brown or brown. For the negative control, PBS was used to replace the primary antibody.

According to the intensity and range of PAX8 expression, the present study divided PEOC with PAX8 expression into 5 categories: No staining was assigned as negative (Fig. 1A and B); $1-24 \%$ staining as $1+$ (Fig. 1C and D); $25-50 \%$ staining as 2+ (Fig. 1E and F); $51-75 \%$ staining as $3+$ (Fig. $1 \mathrm{G}$ and $\mathrm{H}$ ); $>75 \%$ staining as $4+$ (Fig. $1 \mathrm{I}$ and $\mathrm{J}$ ).

The categorization criteria were set as follows: Each slice was observed in 5 independent images, then 100 cells were counted per image and the percentage of stained cells in each slice was calculated.

The aforementioned results were obtained via a double-blind method. Each section was examined by 2 pathologists and the patients with consistent results were enrolled in the present study.
Table I. Expression of PAX8 in ovarian tumor lesions.

\begin{tabular}{lrc}
\hline Tumor & $\begin{array}{c}\text { Patient, } \\
\mathrm{n}\end{array}$ & $\begin{array}{c}\text { Expression of } \\
\text { PAX8, n (\%) }\end{array}$ \\
\hline Primary epithelial ovarian cancer & 60 & $57 / 60(92.00)$ \\
Serous carcinoma & 44 & $42 / 44(95.45)$ \\
Mucinous carcinoma & 9 & $8 / 9(88.89)$ \\
Endometrial carcinoma & 2 & $2 / 2(100.00)$ \\
Clear cell carcinoma & 5 & $5 / 5(100.00)$ \\
Metastatic ovarian carcinoma & 10 & $0 / 10(0.00)$ \\
From gastric cancer & 5 & $0 / 10(0.00)$ \\
From colon cancer & 3 & $0 / 10(0.00)$ \\
From breast cancer & 2 & $0 / 10(0.00)$ \\
Benign ovary tumor & 20 & $17 / 20(85.00)$ \\
Endometriosis & 7 & $6 / 7(85.71)$ \\
Teratoma & 5 & $3 / 5(60.00)$ \\
Serous cystadenoma & 8 & $8 / 8(100.00)$ \\
\hline
\end{tabular}

Statistical analysis. SPSS 13.0 (SPSS, Inc., Chicago, IL, USA) was used for statistical analysis. The association between PAX8 and clinicopathological characteristics was tested using a $\chi^{2}$ test for comparisons between groups, and a Mann-Whitney $\mathrm{U}$ test and Kruskal-Wallis $\mathrm{H}$ test for comparisons within the same population. For survival rate analysis, a Kaplan-Meier test was used to compare the differences between groups. $\mathrm{P}<0.05$ was considered to indicate a statistically significant difference.

\section{Results}

PAX8 expression in ovarian tumor lesions. There were 44 patients with ovarian serous carcinoma (73.3\%), 9 patients with mucinous carcinoma (15\%), 2 patients with endometrioid carcinoma (3.3\%) and 5 patients with clear cell carcinoma (8.3\%) within the total 60 patients with PEOC (Fig. 1).

The IHC staining of PAX8 in PEOC had an overall positive rate of $92 \%$ (57/60). By contrast, all MOC samples showed negative staining. Statistical analysis showed a significant difference between the PEOC and MOC groups $(\mathrm{P}<0.001)$. However, no significant difference was observed between the PEOC groups with different pathological types $(\mathrm{P}=0.871)$.

The positive rate of PAX8 in 20 patients with ovarian benign tumor was $85 \%$ (17/20), showing no significant statistical difference in comparison with the PEOC group $(\mathrm{P}=0.761)$. In addition, no significant difference with respect to the positive expression of PAX8 was found among ovarian benign tumors with different pathologies $(\mathrm{P}=0.533)$. All data shown in Table I.

Association between PAX8 expression and clinicopathological characteristics of PEOC. Based on the aforementioned categorization and statistical analysis, the present study revealed that PAX8 expression was associated with the degree of cancer cell differentiation and International Federation of Gynaecological Oncologists (FIGO) stage (3). The higher the 
A
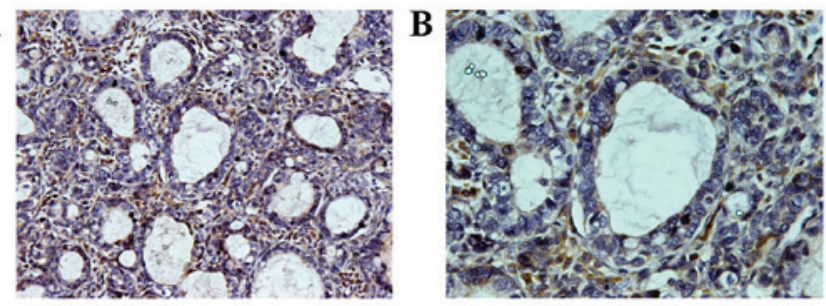

C
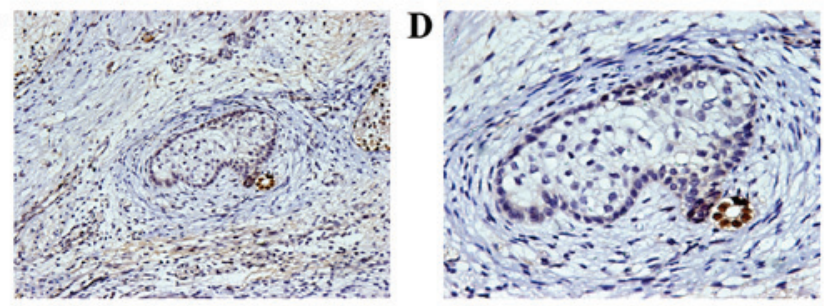

E
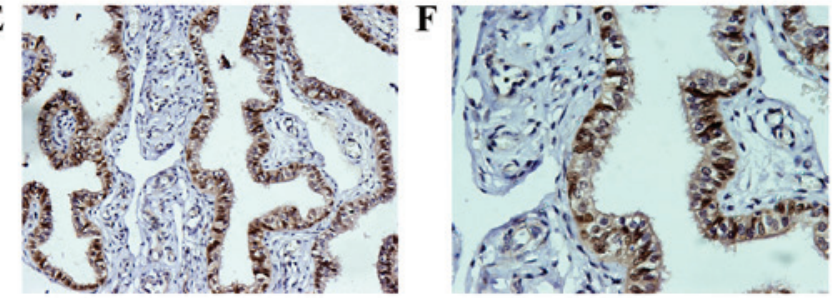

G
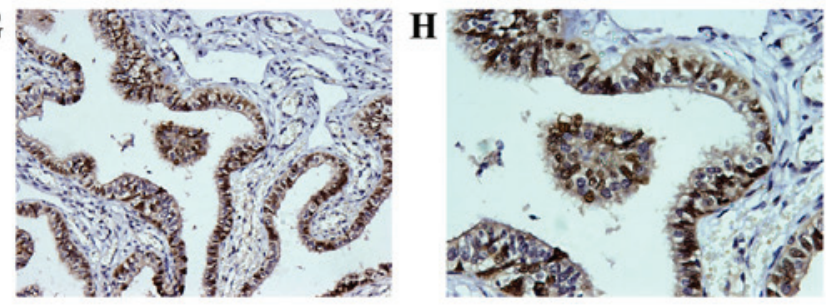

I
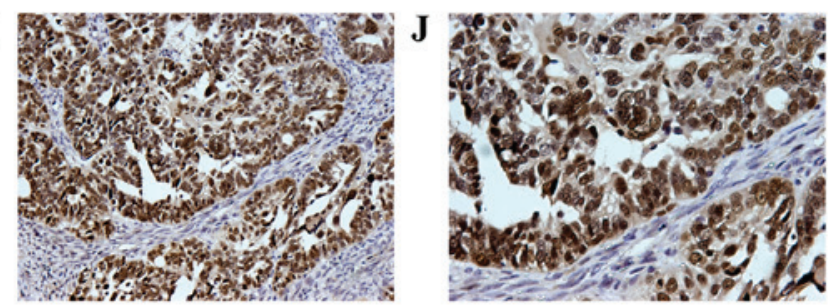

Figure 1. Immunohistochemical staining of PAX8 in ovarian tumors. A PAX8 positive result was taken as the nucleus appearing yellowish-brown or brown. (A) Lesions of metastatic ovarian cancer from gastric adenocarcinoma, with PAX8 negative staining (magnification, x200). (B) Lesions of metastatic ovarian cancer from gastric adenocarcinoma, with PAX8 negative staining (magnification, $\mathrm{x} 400$ ). (C) Lesions of ovarian teratoma, for which PAX8 staining was $1+$ (magnification, $x 200$ ). (D) Lesions of ovarian teratoma, for which PAX8 staining was 1+ (magnification, $x 400)$. (E) Lesions of ovarian serous carcinoma, for which PAX8 staining was 2+ (magnification, x200). (F) Lesions of ovarian serous carcinoma, for which PAX8 staining was 2+ (magnification, $\mathrm{x} 400$ ). (G) Lesions of ovarian serous carcinoma, for which PAX8 staining was 3+ (magnification, $\mathrm{x} 200$ ). (H) Lesions of ovarian serous carcinoma, for which PAX8 staining was 3+ (magnification, $\mathrm{x} 400$ ). (I) Lesions of ovarian serous carcinoma, for which PAX8 staining was 4+ (magnification, $\mathrm{x} 200$ ). (J) Lesions of ovarian serous carcinoma, for which PAX8 staining was 4+ (magnification, $\mathrm{x} 400$ ). PAX8, paired-box 8 .

PAX8 expression, the lower the cancer cell was differentiated $(\mathrm{P}=0.033)$ and the more advanced the FIGO stage was $(\mathrm{P}=0.003$; Table II). There was no significant difference demonstrated between the expression levels of PAX8 in PEOC $(\mathrm{P}=0.574)$ of different pathological types (Table II).

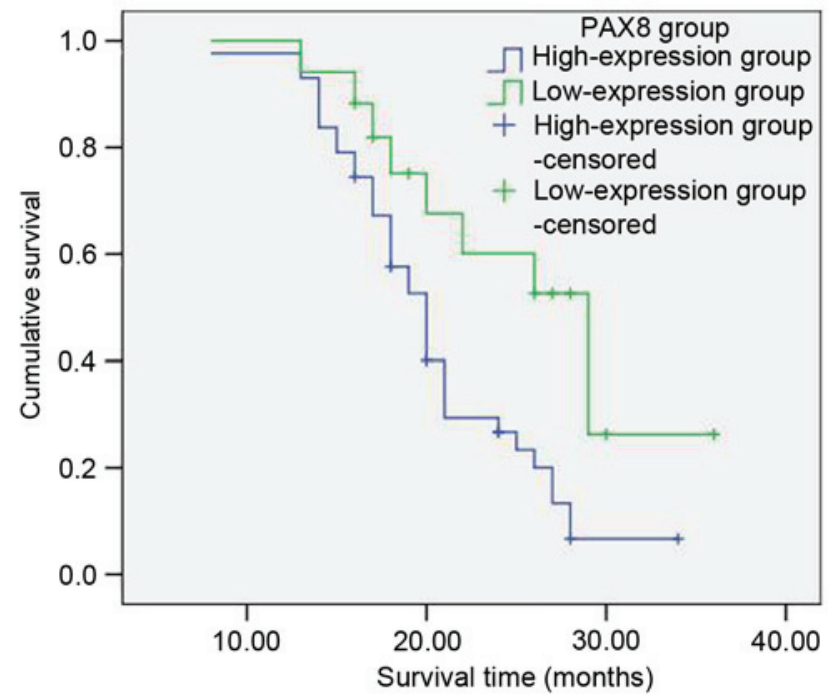

Figure 2. The postoperative survival time of patients with ovarian cancer in the PAX8 low-expression groups was significantly longer than that of the PAX8 high-expression groups. PAX8 staining intensity of negative, $1+$ or $2+$ was classified as PAX8 low expression, while staining intensities of $3+$ and 4+ were classified as PAX8 high expression. PAX8, paired-box 8 .

Association between PAX8 expression and the prognosis of patients with PEOC. Among the group of 60 patients with primary ovarian cancer, the 1-year survival rate was $98 \%$, the 2-year survival rate was 39\% and the 3-year survival rate was $13 \%$. According to the aforementioned categorization based on the PAX8 staining intensity (Table II), with respect to the low PAX8 expression groups (negative, 1+ and 2+; 17 patients in total), the survival rates subsequent to 1,2 and 3 years were $100,60.1$ and $26.3 \%$, respectively. With respect to the high PAX8 groups (3+ and 4+; 43 patients in total), the survival rates subsequent to 1,2 and 3 years were 97.7, 26.7 and $6.7 \%$, respectively. The postoperative survival rate of patients with ovarian cancer in the PAX8 low-expression groups was significantly longer than that of the PAX8 high-expression groups. The difference was considered to be statistically significant $\left(\chi^{2}=6.820, P=0.009\right.$; Table III; Fig. 2).

\section{Discussion}

PAX8 belongs to the paired-box (PAX) gene family. The PAX protein family (PAX 1-9) has a highly conserved region formed of 128 amino acids. The paired box gene family is able to recognize the specific DNA binding site of the target genes to regulate gene expression (13). PAX8 proteins are composed of 450 amino acids located in the 2q12-14 section of encoding genes with a molecular weight of $48 \mathrm{~kb}$ (14). PAX8 was first reported to be involved in thyroid gland development in mice $(15,16)$. During embryonic development, PAX8 performs a key role in the development of organs such as the thyroid, kidney, part of the central nervous system, inner ear, eye and Wolffian and Müllerian ducts (17). Furthermore, PAX8 performs a role in maintaining the normal function of cells subsequent to the birth of the fetus. According to several studies, PAX8 can be a sensitive marker for thyroid, kidney, Müllerian duct and thymus tumors (10-12). 
Table II. Association between PAX8 expression and clinicopathological characteristics of primary epithelial ovarian cancer.

\begin{tabular}{|c|c|c|c|c|c|c|c|c|}
\hline \multirow{2}{*}{$\begin{array}{l}\text { Clinical } \\
\text { characteristic }\end{array}$} & \multirow[b]{2}{*}{ Patient, $\mathrm{n}$} & \multicolumn{5}{|c|}{ PAX8 } & \multirow[b]{2}{*}{$\chi^{2}$} & \multirow[b]{2}{*}{$\mathrm{P}$-value } \\
\hline & & Negative & $1+$ & $2+$ & $3+$ & $4+$ & & \\
\hline Pathological Characteristic & & & & & & & 0.317 & 0.574 \\
\hline Serous carcinoma & 44 & 2 & 4 & 7 & 13 & 18 & & \\
\hline Non-serous carcinoma & 16 & 1 & 1 & 2 & 8 & 4 & & \\
\hline Differentiation degree & & & & & & & 6.850 & 0.033 \\
\hline High & 11 & 1 & 1 & 5 & 3 & 1 & & \\
\hline Moderate & 23 & 2 & 3 & 0 & 8 & 10 & & \\
\hline Low & 26 & 0 & 1 & 4 & 10 & 11 & & \\
\hline FIGO stage & & & & & & & 15.607 & 0.003 \\
\hline I & 9 & 2 & 2 & 2 & 1 & 2 & & \\
\hline II & 17 & 1 & 2 & 5 & 7 & 2 & & \\
\hline III-IV & 34 & 0 & 1 & 2 & 13 & 18 & & \\
\hline
\end{tabular}

FIGO, International Federation of Gynaecological Oncologists.

Table III. Influence of clinicopathological characteristics on the prognosis of 60 patients with primary epithelial ovarian cancer.

\begin{tabular}{|c|c|c|c|c|c|c|}
\hline Characteristic & Patient, $\mathrm{n}$ & $\begin{array}{c}\text { 1-year survival } \\
\text { rate, } \%\end{array}$ & $\begin{array}{c}\text { 2-year survival } \\
\text { rate, } \%\end{array}$ & $\begin{array}{c}\text { 3-year survival } \\
\text { rate, } \%\end{array}$ & $\chi^{2}$ & P-value \\
\hline \multicolumn{7}{|l|}{ PAX8 } \\
\hline$-/ 1+/ 2+$ & 17 & 100 & 60.1 & 26.3 & 6.820 & 0.009 \\
\hline $3+/ 4+$ & 43 & 97.7 & 26.7 & 6.7 & & \\
\hline \multicolumn{7}{|l|}{ Pathological characteristic } \\
\hline Serous carcinoma & 44 & 97.7 & 29.7 & 0 & 2.920 & 0.087 \\
\hline Non-serous carcinoma & 16 & 100 & 50 & 23.4 & & \\
\hline \multicolumn{7}{|l|}{ Differentiation degree } \\
\hline High & 11 & 100 & 42.4 & 0 & 1.566 & 0.457 \\
\hline Moderate & 23 & 100 & 44.3 & 25.9 & & \\
\hline Low & 26 & 96.2 & 24.1 & 6 & & \\
\hline \multicolumn{7}{|l|}{ FIGO stage } \\
\hline $\mathrm{I}-\mathrm{II}$ & 26 & 100 & 56.4 & 33.8 & 10.428 & 0.001 \\
\hline III-IV & 34 & 97.1 & 21.7 & 0 & & \\
\hline
\end{tabular}

FIGO, International Federation of Gynaecological Oncologists; PAX8, paired-box 8.

The female reproductive tract originates from the fetal Müllerian system and PAX8 is involved in the regulation of the formation of the tract (17). Female mice lacking PAX8 expression cannot form the genital tract, leading to sterility (18). For humans, PAX8 is expressed in the tissues and organs, including in secretory cells, derived from the Müllerian system. Additionally, PAX8 expression is found in tumors derived from the epithelial cells of the Müllerian system $(12,19)$. Previous studies have revealed that PAX8 is widely expressed in various types of ovarian tumor, particularly in serous tumors such as ovarian endometrioid carcinoma and clear cell carcinoma $(11,12)$. The majority of serous ovarian cancer cells are considered to originate from the normal secretory cells of the fallopian tube in which PAX8 is highly expressed. Mucinous carcinoma of the Müllerian system is distinguished from metastatic cancer of the digestive tract, and cytokeratin (CK)-7 and CK20 are found to perform an important role in the differentiation process (20). However, in some patients it is difficult to judge the cancer source based on CK7/20 positivity or negativity (20). In certain types of mucinous carcinoma in the female reproductive tract, PAX8 may be partially expressed (21). However, PAX8 is not expressed in carcinoma of the stomach, colon, bile duct, duodenal ampulla, hepatocellular or pancreatic acinar cell carcinoma, and is rarely expressed in adenocarcinoma, pancreas-pseudopapillary tumors of the pancreas or esophageal adenocarcinoma (21). Where PAX8 is expressed, 
metastatic tumors of the digestive tract may be excluded, so the source of PAX8 may be considered to derive from the Müllerian duct. Similarly, PAX8 can be used for the clinical differential diagnosis of breast and ovarian cancer, ovarian sex cord-stromal tumor and malignant mesothelioma $(22,23)$.

In the present study, PAX8 was found to be highly expressed in PEOC, but not in MOC, suggesting that PAX8 exhibits a high enough sensitivity and specificity to be used in clinical practice. In addition, the present study found that PAX8 expression levels are associated with cancer cell differentiation degree and FIGO stage. Survival rate analysis demonstrated that patients with high levels of PAX8 expression had a poor prognosis. The results of the present suggest that there may be a link between PAX8 and the progression of ovarian cancer.

In conclusion, PAX8 IHC detection exhibits high sensitivity and specificity in terms of differentiating between PEOC and MOC. The level of PAX8 expression is associated with the differentiation degree of ovarian tumor cells and FIGO staging, which can help to determine prognosis. However, determining PAX8 expression cannot be used to discriminate between benign and malignant ovarian tumors.

\section{Acknowledgements}

The authors would like to thank Dr Guang-ye Du for the pathological examinations.

\section{References}

1. Sopik V, Lgbal J, Rosen B and Narod SA: Why have ovarian cancer mortality rates declined? Part I. incidence. Gynecol Oncol 138: 741-749, 2015.

2. Jemal A, Tiwari RC, Murray T, Ghafoor A, Samuels A, Ward E, Feuer EJ and Thun MJ; American Cancer Society: Cancer statistics, 2004. CA Cancer J Clin 54: 8-29, 2004.

3. Morgan RJ Jr, Alvarez RD, Armstrong DK, Burger RA, Chen LM, Copeland L, Crispens MA, Gershenson DM, Gray HJ, Hakam A, et al; National comprehensive cancer networks: Ovarian cancer, version 2.2013. J Natl Compr Canc Netw 11: 1199-1209, 2013.

4. Matias-Guiu X and Davidson B: Prognostic biomarkers in endometrial and ovarian carcinoma. Virchows Arch 464: 315-331, 2014.

5. Horn LC, Einenkel J, Handzel R and Höhn AK: Morphology of secondary ovarian tumors and metastases. Pathologe 35: 336-347, 2014 (In German).

6. Yonemura Y, Canbay E, Endou Y, Ishibashi H, Mizumoto A, Miura M, Li Y, Liu Y, Takeshita K, Ichinose M, et al: Peritoneal cancer treatment. Expert Opin Pharmacother 15: 623-636, 2014.

7. Young RH: From krukenberg to today: The ever present problems posed by metastatic tumors in the ovary: Part I. Historical perspective, general principles, mutinous tumors including the krukenberg tumor. Adv Anat Pathol 13: 205-227, 2006.
8. Zhu W and Michael CW: WT1, monoclonal CEA. TTF1, and CA125 antibodies in the differential diagnosis of lung, breast, and ovarian adenocarcinomas in serous effusions. Diagn Cytopathol 35: 370-375, 2007.

9. Lee BH, Hecht JL, Pinkus JL and Pinkus GS: WT1, estrogen receptor, and progesterone receptor as markers for breast or ovarian primary sites in metastatic adenocarcinoma to body fluids. Am J Clin Pathol 117: 745-750, 2002.

10. Ozcan A, Steven SS, Hamilton C, Anjana K, Coffey D, Krishnan B and Truong LD: PAX 8 expression in non-neoplastic tissues, primary tumors, and metastatic tumors: A cornprehensive immunohistochemical study. Mod Pathol 24: 751-764, 2011.

11. Nonaka D, Chiriboga L and Soslow RA: Expression of pax8 as a useful marker in distinguishing ovarian carcinomas from mammary carcinomas. Am J Surg Pathol 32: 1566-1571, 2008.

12. Tong GX, Devaraj K, Hamele-Bena D, Yu WM, Turk A, Chen X, Wright JD and Greenebaum E: PAX8: A marker for carcinoma of Müllerian origin in serous effusions. Diagn Cytopathol 39: 567-574, 2011.

13. Mansouri A, Goudreau G and Gruss P: PAX genes and their role in organogenesis. Cancer Res 59 (7 Suppl): S1707-S1710, 1999.

14. Lang D, Powell SK, Plummer RS, Young KP and Ruggeri BA: PAX genes: Roles in development, pathophysiology, and cancer. Biochem Pharmacol 73: 1-14, 2007.

15. Plachov D, Chowdhury K, Walther C, Simon D, Guenet JL and Gruss P: Pax8, a murine paired box gene expressed in the developing excretory system and thyroid gland. Development 110: 643-651, 1990.

16. Poleev A, Fickenscher H, Mundlos S, Winterpacht A, Zabel B, Fidler A, Gruss P and Plachov D: PAX8, a human paired box gene: Isolation and expression in developing thyroid, kidney and Wilms' tumors. Development 116: 611-623, 1992.

17. Bouchard M, de Caprona D, Busslinger M, Xu P and Fritzsch B: Pax2 and Pax8 cooperate in mouse inner ear morphogenesis and innervation. BMC Dev Biol 10: 89, 2010.

18. Mittag J, Winterhager E, Bauer K and Grümmer R: Congenital hypothyroid female pax8-deficient mice are infertile despite thyroid hormone replacement therapy. Endocrinology 148: 719-725, 2007.

19. Levanon K, Ng V, Piao HY, Zhang Y, Chang MC, Roh MH, Kindelberger DW, Hirsch MS, Crum CP, Marto JA and Drapkin R: Primary ex vivo cultures of human fallopian tube epithelium as a model for serous ovarian carcinogenesis. Oncogene 29: 1103-1113, 2010.

20. Vang R, Gown AM, Barry TS, Wheeler DT, Yemelyanova A, Seidman JD and Ronnett BM: Cytokeratins 7 and 20 in primary and secondary mucinous tumors of the ovary: Analysis of coordinate immunohistochemical expression profiles and staining distribution in 179 cases. Am J Surg Pathol 30: 1130-1139, 2006.

21. Ordóñez NG: Value of PAX 8 immunostaining in tumor diagnosis: A review and update. Adv Anat Pathol 19: 140-151, 2012.

22. Ordóñez NG: Value of PAX8, PAX2, claudin-4, and h-caldesmon immunostaining in distinguishing peritoneal epithelioid mesotheliomas from serous carcinomas. Mod Pathol 26: 553-562, 2013.

23. Laury AR, Hornick JL, Perets R, Krane JF, Corson J, Drapkin R and Hirsch MS: PAX8 reliably distinguishes ovarian serous tumors from malignant mesothelioma. Am J Surg Pathol 34: 627-635, 2010. 